\title{
COMPUTATIONAL ASPECTS OF THE GRAVITATIONAL INSTABILITY PROBLEM FOR A MULTICOMPONENT COSMOLOGICAL MEDIUM
}

\author{
H.J. Haubold ${ }^{1}$ and A.M. Mathai ${ }^{2}$ \\ ${ }^{1}$ Office for Outer Space, United Nations, New York, N.Y., USA \\ ${ }^{2}$ Department of Mathematics and Statistics, McGill University, \\ Montreal, P.Q., Canada H3A 2K6
}

Received... 
Summary. The paper presents results for deriving closed-form analytic solutions of the non-relativistic linear perturbation equations, which govern the evolution of inhomogeneities in a homogeneous spatially flat multicomponent cosmological model. Mathematical methods to derive computable forms of the perturbations are outlined.

Key words: cosmology - growth of perturbation - mathematical methods 


\section{Introduction}

This paper deals with solutions of a system of differential equations describing the evolution of density perturbations in the Universe. Before being more specific about the differential equations, we look at previous work setting the physical environment for deriving such equations.

To formulate a quantitative picture of the evolution of perturbations outside the horizon it is necessary to solve the perturbed EINSTEIN equations. The density contrast $\delta(\vec{x})$ is not a gauge invariant quantity. For sub-horizon-sized perturbations, $a \lambda=\lambda_{\text {phys }} \ll H^{-1}$, this fact is of little consequence, as a NEWTONian analysis is sufficient; $a$ denotes the scale factor and $H$ the HUBBLE parameter, respectively. For super-horizon-sized perturbations, $\lambda_{\text {phys }} \gg H^{-1}$, the gauge transformation for $\delta(\vec{x})$ must be taken into account. In this case a full general relativistic treatment is required. So far two approaches are available for finally deriving the differential equation governing the growth or decay of gravitational instabilities in an expanding Universe. The metric perturbation approach was invented by LIFSHITZ (1946) with his derivation of the synchronous equations. This approach was later used by BARDEEN (1980) to derive the comoving equations. HAWKING (1966) initiated an alternative approach to follow the evolution of the density perturbations in the expanding Universe by employing the general relativistic fluid flow equations. This method was used by OLSON (1976) to derive equations which are equivalent to the synchronous equations, and the same method was further developed by LYTH and MUKHERJEE (1988) to provide the comoving equations.

In order to split up the energy density at a given point in spacetime into an average value plus a perturbation, one must specify a spacelike hypersurface on which the averaging is to be performed. The choice of coordinates in order to define the energy perturbation throughout spacetime is referred to as a choice of gauge (BARDEEN 1980). BARDEEN proposed to use the comoving gauge to deal with the general relativistic equations. Another choice is to use the synchronous gauge (PEEBLES 1980) which, however, is beset by the problem of introducing arbitrary "extra gauge mode" solutions. Actually there is an infinity of synchronous gauges.

A choice of LIFSHITZ's or HAWKING's approach and synchronous or comoving gauge having been made sets the framework for deriving differential equations describing the time evolution of the energy density perturbation. Here again one has the choice to subscribe to the perfect fluid approximation or the kinetic description (PEEBLES 1980). In the perfect fluid approximation, which is more physically transparent, the time evolution of the energy density perturbation is specified by a differential equation involving the pressure perturbation as an additional unknown. The equation is of second order in the comoving gauge, assuming that the pressure perturbation is known, and it has two solutions revealing a growing and decaying mode (PEEBLES 1980). Since the equation governing the amplitude $\delta(t)$ is of second order, there are two solu- 
tions. A given perturbation is expressed as a linear combination of $\delta_{+}(t)$ and $\delta_{-}(t)$. At late times, only the projection onto the growing mode may be important. Physically, the decaying mode corresponds to a perturbation with initial overdensity and velocity arranged so that the initial velocity perturbation eventually "undoes" the density perturbation. In synchronous gauge the equation is of third or higher order corresponding to the extra freedom of choosing the respective gauge.

Many efforts have been made to derive approximate solutions of the equations of relativistic and non-relativistic perturbation theory, usually valid for early time large-scale and for late time small- scale inhomogeneities, respectively. the primary purpose of this paper is to derive closed-form analytic solutions of the non- relativistic linear perturbation equations, which govern the evolution of inhomogeneities in homogeneous spatially flat multicomponent cosmological models. The mathematical method employed here can also be used to tackle the relativistic version of the equations involved, which will be done in a subsequent paper. These closed-form solutions are valid for irregularities on scales smaller than the horizon. They may be used for analytical interpolution between known expressions for short and long wavelength perturbations. In Section 2 we present the physical parameters relevant to the problem and the fundamental differential equations for arbitrary polytropic index $\gamma_{i}$ and a radiation or matter dominated Universe, respectively. Solutions of this fundamental equation are provided in all subsequent Sections 3,4, and 5 for relevant parameter sets including the polytropic index $\gamma_{i}$ and the expansion law index $\eta$. To catalogue these solutions in closed-form, in all Secitons the theory of MEIJER's G- function will be employed. The following results are a continuation of the ones by HAUBOLD, MATHAI, and MUECKET (1991) MATHAI (1989), and NURGALIEV (1986).

\section{Some Parameter of Physical Significance}

The growth of density inhomogeneities can begin as soon as the Universe is matter-dominated. This would be also the case in a Universe dominated by non-interacting relic WIMPs. Baryonic inhomogeneities cannot begin to grow until after decoupling because until then, baryons are tightly coupled to the photons. After decoupling, when the Universe is matter-dominated and baryons are free of the pressure support provided by photons, density inhomogeneities in the baryons and any other matter components can grow. Actually the time of matter-radiation equality is the initial epoch for structure formation. In order to fill in the details of structure formation one needs "initial data" for that epoch. The initial data required include, among others, (1) the total amount of non-relativistic matter in the Universe, quantified by $\Omega_{0}$, and (2) the composition of the Universe, as quantified by the fraction of critical density, $\Omega_{i}=\rho_{i} / \rho_{c}$, contributed by various components of primordial density perturbations ( $\mathrm{i}=$ baryons, WIMPs, relativistic particles, etc.). Here the critical 
density $\rho_{c}$ is the total matter density of the EINSTEIN-DE SITTER Universe. Speculations about the earliest history of the Universe have provided hints as to the appropriate initial data: $\Omega_{0}=1$ from inflation; $0.015 \leq \Omega_{B} \leq 0.15$ and $\Omega_{W I M P} \sim 0.9$ from inflation, primordial nucleosynthesis, and dynamical arguments. In the following we assume that $\sum \Omega_{i}=1, \Omega_{i}=$ const.. The cosmological medium is considered to be unbounded and that there may be a uniform background of relativistic matter. It is further assumed that the matter is only slightly perturbed from the background cosmological model. This assumption may give a good description of the behavior of matter on large scales even when there may be strongly nonlinear clustering on small scales (primordial objects). Also assumed is that matter can be approximated as an ideal fluid with pressure a function of density alone. It consists of $i$ components having the densities $\rho_{i}$ and velocities of sound $\beta_{i}^{2}=d P_{i} / d \rho_{i} \propto \rho^{\gamma_{i}-1}$, when an equation of state

$$
P_{i} \propto \rho^{\gamma_{i}}
$$

has been taken into account. The $i$ components of the medium are interrelated through NEWTON's field equation $\Delta \phi=4 \pi G \sum \rho_{i}$, containing the combined density $\sum \rho_{i}$ of all components. Superimposed upon an initially homogeneous and stationary mass distribution shall be a small perturbation, represented by a sum of plane waves

$$
\delta=\frac{\delta \rho_{i}}{\rho}=\delta_{i}(t) e^{i k x},
$$

where $\lambda=2 \pi a / k$ defines the wave number $k$ and $\lambda$ is the proper wavelength. In the linear approach the system of second order differential equations describing the evolution of the perturbation in the non-relativistic component $i$ is

$$
\frac{d^{2} \delta_{i}}{d t^{2}}+2\left(\frac{\dot{a}}{a}\right) \frac{d \delta_{i}}{d t}+k^{2} \beta_{i}^{2} \delta_{i}=4 \pi G \sum_{j=1}^{m} \rho_{j} \delta_{j}, i=1, \ldots, m
$$

where for all perturbations the same wave number $\mathrm{k}$ is used. This equation is valid for all sub-horizon-sized perturbations in any non-relativistic species, so long as the usual FRIEDMANN equation for the expansion rate is used. Although the following investigation of closed-form solutions of the fundamental differential equation governing the evolution of inhomogeneities in a multicomponent cosmological medium is quite general, it will be presented within the context of the inflationary scenario. Therefore in the following we consider an EINSTEIN-DE SITTER Universe with zero cosmological constant. After inflation the FRIEDMANN equation for the cosmological evolution reduces to

$$
\left(\frac{\dot{a}}{a}\right)^{2}=H^{2}=\frac{8 \pi G}{3} \rho .
$$

The continuity equation gives $\rho \propto a^{-3}$ and from eq. (4) one has $a \propto t^{2 / 3}$ and $\rho_{i}=\Omega_{i} / 6 \pi G t^{2}$. During the expansion the HUBBLE parameter changes as 
$H=\eta t^{-1}$, where the expansion law index is $\eta=\frac{1}{2}$ in the radiation-dominated epoch and $\eta=\frac{2}{3}$ in the matter-dominated epoch respectively. The wave number is proportional to $a^{-1}$ so that $k^{2} \beta_{i}^{2}=k_{i}^{2} t^{2\left(1-\eta-\gamma_{i}\right)}$ in eq. (3), where now the constants $k_{i}$ come from both the wave vector and the velocity of sound. If $k_{i}=$ 0 the sound velocity $\beta_{i}$ equals zero, in which case the adiabatic index $\gamma_{i}$ loses its sense. Defining the parameter $\alpha_{i}=2\left(2-\eta-\gamma_{i}\right)$ that absorbs the adiabatic index as well as the type of expansion law we can write for eq. (3) using eq. (4):

$$
t^{2} \ddot{\delta}_{i}(t)+2 \eta t \dot{\delta}_{i}(t)+k_{i}^{2} t^{\alpha_{i}} \delta_{i}=\frac{2}{3} \sum_{j=1}^{m} \Omega_{j} \delta_{j}(t), \quad i=1, \ldots, m,
$$

and dots denote derivatives with respect to time. We introduce a time operator $\Delta=t \mathrm{~d} / \mathrm{d} t$ and change the dependent variable, the density perturbation $\delta_{i}(t)$, dealing with the function $\Phi_{i}$ instead of $\delta_{i}$ by setting $\delta_{i}(t)=t^{\alpha} \Phi_{i}(t)$. The equation (5) for the function $\Phi_{i}$ is then given by

$$
\Delta^{2} \Phi_{i}+b_{i} \Phi_{i}=\frac{2}{3} \sum_{j=1}^{m} \Omega_{j} \Phi_{j}
$$

where

$$
\Phi_{i}=t^{-\alpha} \delta_{i}, b_{i}=k_{i}^{2} t^{\alpha_{i}}-\alpha^{2}, \alpha_{i}=2\left(2-\eta-\gamma_{i}\right), \alpha=-\left(\frac{2 \eta-1}{2}\right) .
$$

Observing that $\Sigma \Omega_{j}=1$ and operating on both sides of the differential equation for the $\Phi_{i}$ by $\Delta^{2}$ we obtain the fundamental equation

$$
\Delta^{4} \Phi_{i}+\Delta^{2}\left(b_{i} \Phi_{i}\right)-\frac{2}{3}\left(\Delta^{2} \Phi_{i}+b_{i} \Phi_{i}\right)=-\frac{2}{3} \sum_{j=1}^{m} b_{j} \Omega_{j} \Phi_{j}, \quad i=1, \ldots m,
$$

(Mathai, 1989). As indicated above for $\eta$ the values $\frac{2}{3}$ and $\frac{1}{2}$ are significant and in what follows it will be assumed that $2 \geq \gamma_{i} \geq \frac{2}{3}$. We have chosen the range of values of $\gamma_{i}$ for physical as well as mathematical reasons as will be evident later in the analysis of eq. (7). For some of these parameter values we will consider a multicomponent medium. Consider the special case

$$
\begin{aligned}
& b_{1}=k_{1}^{2} t^{\alpha_{1}}-\frac{(2 \eta-1)^{2}}{4}, \\
& b_{2}=b_{3}=\cdots=b_{m}=b=k^{2} t^{\alpha}-\frac{(2 \eta-1)^{2}}{4}
\end{aligned}
$$

of the fundamental equation (7). This is the case considered in Haubold, Mathai and Muecket (1991) in detail. In the present discussion we will use the same notations as in Haubold, Mathai and Muecket (1991). Let $k=0$ in (8). This is 
case 4.1 of Haubold, Mathai and Muecket (1991). For this case the parameters are the following:

$$
\begin{gathered}
b_{1}^{*}, b_{2}^{*}= \pm\left\{\frac{(2 \eta-1)^{2}}{4 \alpha_{1}^{2}}\right\}^{\frac{1}{2}}, b_{3}^{*}, b_{4}^{*}= \pm\left\{\frac{1}{\alpha_{1}^{2}}\left[\frac{(2 \eta-1)^{2}}{4}+\frac{2}{3}\right]\right\}^{\frac{1}{2}}, \\
a_{1}^{*}, a_{2}^{*}=-1 \pm\left\{\frac{1}{\alpha_{1}^{2}}\left[\frac{(2 \eta-1)^{2}}{4}+\frac{2}{3}-\frac{2}{3} \Omega_{1}\right]\right\}^{\frac{1}{2}} .
\end{gathered}
$$

Table 2.1 gives these parameters for $\eta=\frac{2}{3}, \frac{1}{2}$ and $\gamma_{i}=\frac{2}{3}, 1, \frac{4}{3}, \frac{5}{3}, 2$. 
Table 2.1

\begin{tabular}{l|l|l|l|l|l}
\hline$\eta, \gamma_{i}$ & $\alpha_{i}$ & $\alpha$ & $b_{1}^{*}, b_{2}^{*}$ & $b_{3}^{*}, b_{4}^{*}$ & $a_{1}^{*}, a_{2}^{*}$ for $\Omega_{1}=\frac{1}{2}$ \\
\hline \hline$\frac{2}{3} \frac{4}{3}$ & 0 & $-\frac{1}{6}$ & & & \\
\hline$\frac{2}{3} 1$ & $\frac{2}{3}$ & $-\frac{1}{6}$ & $\pm \frac{1}{4}$ & $\pm \frac{5}{4}$ & $-1 \pm \frac{\sqrt{13}}{4}$ \\
\hline$\frac{2}{3} \frac{2}{3}$ & $\frac{4}{3}$ & $-\frac{1}{6}$ & $\pm \frac{1}{8}$ & $\pm \frac{5}{8}$ & $-1 \pm \frac{\sqrt{13}}{8}$ \\
\hline$\frac{2}{3} \frac{5}{3}$ & $-\frac{2}{3}$ & $-\frac{1}{6}$ & $\pm \frac{1}{4}$ & $\pm \frac{5}{4}$ & $-1 \pm \frac{\sqrt{13}}{4}$ \\
\hline$\frac{2}{3} 2$ & $-\frac{4}{3}$ & $-\frac{1}{6}$ & $\pm \frac{1}{8}$ & $\pm \frac{5}{8}$ & $-1 \pm \frac{\sqrt{13}}{8}$ \\
\hline$\frac{1}{2} \frac{4}{3}$ & $\frac{1}{3}$ & 0 & 0 & $\pm \sqrt{6}$ & $-1 \pm \sqrt{3}$ \\
\hline$\frac{1}{2} 1$ & 1 & 0 & 0 & $\pm \sqrt{\frac{2}{3}}$ & $-1 \pm \frac{1}{\sqrt{3}}$ \\
\hline$\frac{1}{2} \frac{2}{3}$ & $\frac{5}{3}$ & 0 & 0 & $\pm \frac{\sqrt{6}}{5}$ & $-1 \pm \frac{\sqrt{3}}{5}$ \\
\hline$\frac{1}{2} \frac{5}{3}$ & $-\frac{1}{3}$ & 0 & 0 & $\pm \sqrt{6}$ & $-1 \pm \sqrt{3}$ \\
\hline$\frac{1}{2} 2$ & -1 & 0 & 0 & $\pm \sqrt{\frac{2}{3}}$ & $-1 \pm \frac{1}{\sqrt{3}}$ \\
\hline
\end{tabular}

When evaluating the solutions explicitly one needs the parameter differences.

These are given in Table 2.2.

Table 2.2

\begin{tabular}{l|l|l|l|l|l|l|l|l|l|l|l}
\hline$\eta$ & $\gamma_{i}$ & $b_{1}^{*}$ & $b_{2}^{*}$ & $b_{3}^{*}$ & $b_{4}^{*}$ & $b_{1}^{*}-b_{2}^{*}$ & $b_{1}^{*}-b_{3}^{*}$ & $b_{1}^{*}-b_{4}^{*}$ & $b_{2}^{*}-b_{3}^{*}$ & $b_{2}^{*}-b_{4}^{*}$ & $b_{3}^{*}-b_{4}^{*}$ \\
\hline \hline$\frac{2}{3}$ & 1 & $\frac{1}{4}$ & $-\frac{1}{4}$ & $\frac{5}{4}$ & $-\frac{5}{4}$ & $\frac{1}{2}$ & -1 & $\frac{3}{2}$ & $-\frac{3}{2}$ & 1 & $\frac{5}{2}$ \\
$\frac{2}{3}$ & $\frac{2}{3}$ & $\frac{1}{8}$ & $-\frac{1}{8}$ & $\frac{5}{8}$ & $-\frac{5}{8}$ & $\frac{1}{4}$ & $-\frac{1}{2}$ & $\frac{3}{4}$ & $-\frac{3}{4}$ & $\frac{1}{2}$ & $\frac{5}{4}$ \\
$\frac{2}{3}$ & $\frac{5}{3}$ & $\frac{1}{4}$ & $-\frac{1}{4}$ & $\frac{5}{4}$ & $-\frac{5}{4}$ & $\frac{1}{2}$ & -1 & $\frac{3}{2}$ & $-\frac{3}{2}$ & 1 & $\frac{5}{2}$ \\
$\frac{2}{3}$ & 2 & $\frac{1}{8}$ & $-\frac{1}{8}$ & $\frac{5}{8}$ & $-\frac{5}{8}$ & $\frac{1}{4}$ & $-\frac{1}{2}$ & $\frac{3}{4}$ & $-\frac{3}{4}$ & $\frac{1}{2}$ & $\frac{5}{4}$ \\
\hline \hline$\frac{1}{2}$ & $\frac{4}{3}$ & 0 & 0 & $\sqrt{6}$ & $-\sqrt{6}$ & 0 & $-\sqrt{6}$ & $\sqrt{6}$ & $-\sqrt{6}$ & $\sqrt{6}$ & $2 \sqrt{6}$ \\
$\frac{1}{2}$ & 1 & 0 & 0 & $\sqrt{\frac{2}{3}}$ & $-\sqrt{\frac{2}{3}}$ & 0 & $-\sqrt{\frac{2}{3}}$ & $\sqrt{\frac{2}{3}}$ & $-\sqrt{\frac{2}{3}}$ & $\sqrt{\frac{2}{3}}$ & $2 \sqrt{\frac{2}{3}}$ \\
$\frac{1}{2}$ & $\frac{2}{3}$ & 0 & 0 & $\frac{\sqrt{6}}{5}$ & $-\frac{\sqrt{6}}{5}$ & 0 & $-\frac{\sqrt{6}}{5}$ & $\frac{\sqrt{6}}{5}$ & $-\frac{\sqrt{6}}{5}$ & $\frac{\sqrt{6}}{5}$ & $2 \frac{\sqrt{6}}{5}$ \\
$\frac{1}{2}$ & $\frac{5}{3}$ & 0 & 0 & $\sqrt{6}$ & $-\sqrt{6}$ & 0 & $-\sqrt{6}$ & $\sqrt{6}$ & $-\sqrt{6}$ & $\sqrt{6}$ & $2 \sqrt{6}$ \\
$\frac{1}{2}$ & 2 & 0 & 0 & $\sqrt{\frac{2}{3}}$ & $-\sqrt{\frac{2}{3}}$ & 0 & $-\sqrt{\frac{2}{3}}$ & $\sqrt{\frac{2}{3}}$ & $-\sqrt{\frac{2}{3}}$ & $\sqrt{\frac{2}{3}}$ & $2 \sqrt{\frac{2}{3}}$ \\
\hline
\end{tabular}




\section{Solution when $\alpha_{1}=0$}

Note that when $\eta=\frac{2}{3}$ and $\gamma_{i}=\frac{4}{3}$ one can have $\alpha_{i}=0$ in equation (6). The exact solution given in equation (5.3) of Haubold, Mathai and Muecket (1991) is for the situation $\alpha_{i} \neq 0$. Hence this case needs separate discussion. When $\alpha_{1}=0$ the fundamental equation (7) reduces to the form

$$
\begin{aligned}
\Delta^{4} \Phi_{1}- & {\left[\frac{(2 \eta-1)^{2}}{2}+\frac{2}{3}-k_{1}^{2}\right] \Delta^{2} \Phi_{1}+\left[\frac{(2 \eta-1)^{4}}{16}+\frac{2}{3} \frac{(2 \eta-1)^{2}}{4}\right.} \\
& \left.+\left(\frac{2}{3} \Omega_{1}-\frac{(2 \eta-1)^{2}}{4}-\frac{2}{3}\right) k_{1}^{2}\right] \Phi_{1}=0 .
\end{aligned}
$$

In this case the general solution is of the form

$$
\Phi_{1}=c_{1} t^{d_{1}}+c_{2} t^{d_{2}}+c_{3} t^{d_{3}}+c_{4}^{d_{4}},
$$

where $c_{1}, c_{2}, c_{3}, c_{4}$ are arbitrary constants and $d_{1}, d_{2}, d_{3}, d_{4}$ are the roots of the equation

$$
\begin{aligned}
x^{4}- & {\left[\frac{(2 \eta-1)^{2}}{2}+\frac{2}{3}-k_{1}^{2}\right] x^{2} } \\
+ & {\left[\frac{(2 \eta-1)^{4}}{16}+\frac{2}{3} \frac{(2 \eta-1)^{2}}{4}+\left\{\frac{2}{3} \Omega_{1}-\frac{(2 \eta-1)^{2}}{4}-\frac{2}{3}\right\} k_{1}^{2}\right]=0 . }
\end{aligned}
$$

They can be seen to be the following:

$$
\begin{aligned}
& d_{1}, d_{2}= \pm\left[\frac{(2 \eta-1)^{2}}{4}+\frac{1}{3}-\frac{k_{1}^{2}}{2}+\left\{\left(\frac{1}{3}+\frac{k_{1}^{2}}{2}\right)-\frac{2}{3} \Omega_{1}\right\}^{\frac{1}{2}}\right]^{\frac{1}{2}} \\
& d_{3}, d_{4}= \pm\left[\frac{(2 \eta-1)^{2}}{4}+\frac{1}{3}-\frac{k_{1}^{2}}{2}-\left\{\left(\frac{1}{3}+\frac{k_{1}^{2}}{2}\right)-\frac{2}{3} \Omega_{1}\right\}^{\frac{1}{2}}\right]^{\frac{1}{2}}
\end{aligned}
$$

\section{Solution when the parameters differ by integers}

The general solution given in Haubold, Mathai and Muecket (1991), equations (5.2) and (5.3), are for finite values of $\mathrm{t}$ and for the cases that the $b_{j}^{*}$ 's do not differ by integers. From Table 2.2 note that at two points, namely $\left(\eta=\frac{2}{3}, \gamma_{i}=\frac{5}{3}\right)$ and $\left(\eta=\frac{2}{3}, \gamma_{i}=1\right)$ one has $b_{1}^{*}-b_{3}^{*}=-1$ and $b_{4}^{*}-b_{2}^{*}=-1$. This means that $G_{1}$ and $G_{4}$ of (5.3) of Haubold, Mathai and Muecket (1991) need modifications. At all other points the $G_{j}$ 's remain the same. 


\subsection{Modifications of the $G_{j}^{\prime} s$ for the cases $\left(\eta=\frac{2}{3}, \gamma_{i}=1\right)$}

$$
\text { and }\left(\eta=\frac{2}{3}, \gamma_{i}=\frac{5}{3}\right)
$$

Consider, for $x=k_{1}^{2} t^{\alpha_{1}} / \alpha_{1}^{2}, \alpha_{1} \neq 0$,

$$
\begin{aligned}
G_{1} & =G_{2,4}^{1,2}\left(\left.x\right|_{b_{1}^{*}, b_{2}^{*}, b_{3}^{*}, b_{4}^{*}} ^{a_{1}^{*}+1, a^{*}+1}\right) \\
& =\frac{1}{2 \pi i} \int_{L} \frac{\Gamma\left(\frac{1}{4}+s\right) \Gamma\left(-a_{1}^{*}-s\right) \Gamma\left(-a_{2}^{*}-s\right) x^{-s} d s}{\Gamma\left(\frac{5}{4}-s\right) \Gamma\left(-\frac{1}{4}-s\right) \Gamma\left(\frac{9}{4}-s\right)} .
\end{aligned}
$$

Note that a zero coming from $\Gamma\left(-\frac{1}{4}-s\right)$ at $s=-\frac{1}{4}$ coincides with a pole coming from $\Gamma\left(\frac{1}{4}+s\right)$ at $s=-\frac{1}{4}$. This can be removed by rewriting as follows:

$$
G_{1}=-\frac{1}{2 \pi i} \int_{L} \frac{\Gamma\left(\frac{5}{4}+s\right) \Gamma\left(-a_{1}^{*}-s\right) \Gamma\left(-a_{2}^{*}-s\right) x^{-s} d s}{\Gamma\left(\frac{5}{4}-s\right) \Gamma\left(\frac{3}{4}-s\right) \Gamma\left(\frac{9}{4}-s\right)} .
$$

Evaluating (16) as the sum of the residues at the poles of $\Gamma\left(\frac{5}{4}+s\right)$ one has

$$
\begin{aligned}
G_{1}= & -x^{\frac{5}{4}} \frac{\Gamma\left(-a_{1}^{*}+\frac{5}{4}\right) \Gamma\left(-a_{2}^{*}+\frac{5}{4}\right)}{\Gamma\left(\frac{10}{4}\right) \Gamma(2) \Gamma\left(\frac{14}{4}\right)} \\
& \times{ }_{2} F_{3}\left(-a_{1}^{*}+\frac{5}{4},-a_{2}^{*}+\frac{5}{4} ; \frac{10}{4}, 2, \frac{14}{4} ;-x\right) .
\end{aligned}
$$

Evaluating $G_{4}$ also the same way one has the following:

$$
\begin{aligned}
G_{4}= & G_{2,4}^{1,2}\left(\left.x\right|_{b_{4}^{*}, b_{1}^{*}, b_{2}^{*}, b_{3}^{*}} ^{a^{*}+1, a^{*}+1}\right) \\
= & \frac{1}{2 \pi i} \int_{L} \frac{\Gamma\left(-\frac{5}{4}+s\right) \Gamma\left(-a_{1}^{*}-s\right) \Gamma\left(-a_{2}^{*}-s\right)}{\Gamma\left(\frac{3}{4}-s\right) \Gamma\left(\frac{5}{4}-s\right) \Gamma\left(-\frac{1}{4}-s\right)} x^{-s} d s \\
= & -\frac{1}{2 \pi i} \int_{L} \frac{\Gamma\left(-\frac{1}{4}+s\right) \Gamma\left(-a_{1}^{*}-s\right) \Gamma\left(-a_{2}^{*}-s\right)}{\Gamma\left(\frac{3}{4}-s\right) \Gamma\left(\frac{9}{4}-s\right) \Gamma\left(-\frac{1}{4}-s\right)} x^{-s} d s \\
= & -x^{-\frac{1}{4}} \frac{\Gamma\left(-\frac{1}{4}-a_{1}^{*}\right) \Gamma\left(-\frac{1}{4}-a_{2}^{*}\right)}{\Gamma\left(\frac{1}{2}\right) \Gamma(2) \Gamma\left(-\frac{1}{2}\right)} \\
& \times{ }_{2} F_{3}\left(-\frac{1}{4}-a_{1}^{*},-\frac{1}{4}-a_{2}^{*} ; \frac{1}{2}, 2,-\frac{1}{2} ;-x\right) .
\end{aligned}
$$

Thus the complete solution in this case is of the form

$$
\Phi_{1}=c_{1} G_{1}+c_{2} G_{2}+c_{3} G_{3}+c_{4} G_{4}
$$

where $c_{1}, c_{2}, c_{3}, c_{4}$ are arbitrary constants, $G_{1}$ and $G_{4}$ are given in (17) and (18) respectively and $G_{2}$ and $G_{3}$ are given in equation (5.3) of Haubold, Mathai and Muecket (1991). 


\section{Solution near $\infty$ for the parameter values of table 2.1}

Except for the points $\left(\eta, \gamma_{i}\right)=\left(\frac{2}{3}, \frac{2}{3}\right),\left(\frac{2}{3}, 2\right)$ in all other cases some of the parameters differ by integers. In five cases of Table 2.2 the poles in the integrand can be up to order 2 and in two cases the poles can be of order up to 3 . The general solution near $\infty$ is of the form

$$
\Phi_{1}=f_{1} F_{1}+f_{2} F_{2}+f_{3} F_{3}+f_{4} F_{4},
$$

where $f_{1}, f_{2}, f_{3}, f_{4}$ are arbitrary constants and $F_{j}$ are given in the equation (5.6) of Haubold, Mathai and Muecket (1991). For example,

$$
F_{1}=G_{2,4}^{4,1}\left(\left.x\right|_{b_{1}^{*}, b_{2}^{*}, b_{3}^{*}, b_{4}^{*}} ^{1+a_{*}^{*}, 1+a_{*}^{*}}\right), x=k_{1}^{2} t^{\alpha_{1}} / \alpha_{1}^{2}, \alpha_{1} \neq 0 .
$$

$F_{2}$ is available from $F_{1}$ by interchanging $a_{1}^{*}$ and $a_{2}^{*} . \quad F_{3}$ and $F_{4}$ are available from $F_{1}$ by replacing $x$ by $x e^{i \pi}$ and $x e^{-i \pi}$ respectively. We will evaluate $F_{1}$ for the parameter values which differ by integers.

$$
\text { 5.1. } F_{1} \text { for }\left(\eta=\frac{1}{2}, \gamma_{i}=\frac{4}{3}\right)
$$

In this case one has

$$
F_{1}=\frac{1}{2 \pi i} \int_{L} \frac{\Gamma(s) \Gamma(s) \Gamma(\sqrt{6}+s) \Gamma(-\sqrt{6}+s) \Gamma\left(-a_{1}^{*}-s\right)}{\Gamma\left(1+a_{2}^{*}+s\right)} x^{-s} d s .
$$

Note that at $s=0,-1,-2, \ldots$ the integrand has poles of order 2 each. All other poles are of order 1 each. Thus

$$
F_{1}=H_{1}+H_{2}+H_{3},
$$

where $H_{2}$ and $H_{3}$ are the sums of the residues at the poles of $\Gamma(\sqrt{6}+s)$ and $\Gamma(-\sqrt{6}+s)$ respectively and $H_{1}$ is the sum of the residues at the poles of $\Gamma^{2}(s)$. For all the cases of simple poles including $\mathrm{H}_{2}$ and $\mathrm{H}_{3}$ one has the result as follows:

$$
\begin{aligned}
R_{j}= & x^{b_{j}^{*}}\left[\Pi_{k=1}^{\prime} \Gamma\left(b_{k}^{*}-b_{j}^{*}\right)\right] \frac{\Gamma\left(-a_{1}^{*}+b_{j}^{*}\right)}{\Gamma\left(1+a_{2}^{*}-b_{j}^{*}\right)} \\
& \times{ }_{2} F_{3}\left(-a_{1}^{*}+b_{j}^{*},-a_{2}^{*}+b_{j}^{*} ; 1-b_{1}^{*}+b_{j}^{*}, \ldots, \#, \ldots, 1-b_{4}^{*}+b_{j}^{*} ;-x\right),
\end{aligned}
$$

where $\Pi^{\prime}$ denotes the absence of the gamma $\Gamma\left(b_{k}^{*}-b_{k}^{*}\right)$ and \# denotes the absence of the element $1-b_{j}^{*}-b_{j}^{*}$. Thus one has,

$$
\begin{aligned}
H_{2}= & x^{\sqrt{6}} \Gamma^{2}(-\sqrt{6}) \Gamma(-2 \sqrt{6}) \frac{\Gamma\left(-a_{1}^{*}+\sqrt{6}\right)}{\Gamma\left(1+a_{2}^{*}-\sqrt{6}\right)} \\
& \times{ }_{2} F_{3}\left(-a_{1}^{*}+\sqrt{6},-a_{2}^{*}+\sqrt{6} ; 1+\sqrt{6}, 1+\sqrt{6}, 1+2 \sqrt{6} ;-x\right)
\end{aligned}
$$


and

$$
\begin{aligned}
H_{3}= & x^{-\sqrt{6}} \Gamma^{2}(\sqrt{6}) \Gamma(2 \sqrt{6}) \frac{\Gamma\left(-a_{1}^{*}-\sqrt{6}\right)}{\Gamma\left(1+a_{2}^{*}+\sqrt{6}\right)} \\
& \times{ }_{2} F_{3}\left(-a_{1}^{*}-\sqrt{6},-a_{2}^{*}-\sqrt{6} ; 1-\sqrt{6}, 1-\sqrt{6}, 1-2 \sqrt{6} ;-x\right) .
\end{aligned}
$$

Now consider the evaluation of $H_{1}$. Note that for all parameter combinations $\left(\eta, \gamma_{i}\right)=\left(\frac{1}{2}, \frac{4}{3}\right),\left(\frac{1}{2}, 1\right),\left(\frac{1}{2}, \frac{2}{3}\right),\left(\frac{1}{2}, \frac{5}{3}\right),\left(\frac{1}{2}, 2\right)$ we have $b_{1}^{*}=0=b_{2}^{*}$. Hence we can write $H_{1}$ in general terms as follows,

$$
H_{1}=\frac{1}{2 \pi i} \int_{L} \frac{\Gamma^{2}(s) \Gamma\left(b_{3}^{*}+s\right) \Gamma\left(b_{4}^{*}+s\right) \Gamma\left(-a_{1}^{*}-s\right)}{\Gamma\left(1+a_{2}^{*}+s\right)} x^{-s} d s .
$$

The general technique of evaluating such integrals is available in Mathai and Saxena (1973). The solution is the following

$$
\begin{aligned}
H_{1}= & \sum_{\nu=0}^{\infty}\left[\frac{1}{(\nu !)^{2}}-(\ln x)\left\{2 \psi(\nu+1)+\psi\left(b_{3}^{*}-\nu\right)+\psi\left(b_{4}^{*}-\nu\right)\right.\right. \\
& \left.\left.+\psi\left(-a_{1}^{*}+\nu\right)-\psi\left(1+a_{2}^{*}-\nu\right)\right\}\right] \\
& \times \Gamma\left(b_{3}^{*}-\nu\right) \Gamma\left(b_{4}^{*}-\nu\right) \frac{\Gamma\left(-a_{1}^{*}+\nu\right)}{\Gamma\left(1+a_{2}^{*}-\nu\right)} \frac{x^{\nu}}{(\nu !)^{2}},
\end{aligned}
$$

where the $b_{3}^{*}$ and $b_{4}^{*}$ are given in Table 2.1 for the specific parameter combinations and $\psi()$ is a psi function. Note that the first part in (27) can be written as a ${ }_{2} F_{3}\left(-a_{1}^{*},-a_{2}^{*} ; 1,1-b_{3}^{*}, 1-b_{4}^{*} ;-x\right)$.

5.2. $F_{1}$ for $\left(\eta=\frac{2}{3}, \gamma_{i}=1\right)$ and $\left(\eta=\frac{2}{3}, \gamma_{i}=\frac{5}{3}\right)$

In these two cases $F_{1}$ is of the following form:

$$
\begin{aligned}
F_{1} & =\frac{1}{2 \pi i} \int_{L} \frac{\Gamma\left(\frac{1}{4}+s\right) \Gamma\left(-\frac{1}{4}+s\right) \Gamma\left(\frac{5}{4}+s\right) \Gamma\left(-\frac{5}{4}+s\right) \Gamma\left(-a_{1}^{*}-s\right) x^{-s} d s}{\Gamma\left(1+a_{2}^{*}+s\right)}(29) \\
& =\frac{1}{2 \pi i} \int_{L} \frac{\Gamma^{2}\left(\frac{5}{4}+s\right) \Gamma^{2}\left(-\frac{1}{4}+s\right) \Gamma\left(-a_{1}^{*}-s\right)}{\left(s+\frac{1}{4}\right)\left(s-\frac{5}{4}\right) \Gamma\left(1+a_{2}^{*}+s\right)} x^{-s} d s .
\end{aligned}
$$

There are poles of order one each at $s=-\frac{1}{4}$ and $s=\frac{5}{4}$ respectively and poles of order 2 each at $s=-\frac{5}{4}-\nu, s=\frac{1}{4}-\lambda, \nu=0,1, \ldots, \lambda=0,1, \ldots$ respectively. Thus $F_{1}$ is evalueated as the sum of the residues at all these poles. Let us denote these residues by $R_{1}, R_{2}, R_{3}$ and $R_{4}$ respectively. Then

$$
F_{1}=R_{1}+R_{2}+R_{3}+R_{4}
$$

where

$$
R_{1}=\text { residue at } s=-\frac{1}{4}
$$




$$
\begin{aligned}
& =-\frac{\Gamma^{2}\left(-\frac{1}{2}\right) \Gamma\left(-a_{1}^{*}+\frac{1}{4}\right)}{\left(\frac{3}{2}\right) \Gamma\left(\frac{3}{4}+a_{2}^{*}\right)} x^{\frac{1}{4}}=-\frac{2}{3} \frac{\Gamma^{2}\left(-\frac{1}{2}\right) \Gamma\left(-a_{1}^{*}+\frac{1}{4}\right)}{\Gamma\left(\frac{3}{4}+a_{2}^{*}\right)} x^{\frac{1}{4}} \\
& R_{2}=\text { residue at } s=\frac{5}{4} \\
& =\frac{2}{3} \frac{\Gamma^{2}\left(\frac{5}{2}\right) \Gamma\left(-a_{1}^{*}+\frac{5}{4}\right)}{\Gamma\left(\frac{9}{4}+a_{2}^{*}\right)} x^{-\frac{5}{4}} \\
& R_{3}=x^{\frac{5}{4}} \sum_{\nu=0}^{\infty}\left\{-\ln x+2 \psi(\nu+1)+2 \psi\left(-\frac{3}{2}-\nu\right)+\psi\left(-a_{1}^{*}+\frac{5}{4}+\nu\right)\right. \\
& \left.-\psi\left(1+a_{2}^{*}-\frac{5}{4}-\nu\right)+\frac{1}{\nu+1}+\frac{1}{\left(\frac{5}{4}+\nu\right)}\right\} \\
& \times \frac{\Gamma^{2}\left(-\frac{3}{2}-\nu\right) \Gamma\left(-a_{1}^{*}+\frac{5}{4}+\nu\right)}{\Gamma\left(1+a_{2}^{*}-\frac{5}{4}-\nu\right)(1+\nu)\left(\frac{5}{2}+\nu\right)} \frac{x^{\nu}}{(\nu !)^{2}} \\
& R_{4}=x^{-\frac{1}{4}} \sum_{\nu=0}^{\infty}\left\{-\ln x+2 \psi(\nu+1)+2 \psi\left(\frac{3}{2}-\nu\right)\right. \\
& \left.+\psi\left(-a_{1}^{*}-\frac{1}{4}+\nu\right)-\psi\left(\frac{5}{4}+a_{2}^{*}-\nu\right)+\frac{1}{\left(-\frac{1}{2}+\nu\right)}+\frac{1}{1+\nu}\right\} \\
& \times \frac{\Gamma^{2}\left(\frac{3}{2}-\nu\right) \Gamma\left(-a_{1}^{*}-\frac{1}{4}+\nu\right)}{\left(\frac{1}{2}-\nu\right)(-1-\nu) \Gamma\left(\frac{5}{4}+a_{2}^{*}-\nu\right)} \frac{x^{\nu}}{(\nu !)^{2}} .
\end{aligned}
$$

As before $F_{2}$ is available from the $F_{1}$ of (30) by interchanging $a_{1}^{*}$ and $a_{2}^{*}$. $F_{3}$ and $F_{4}$ are available from $F_{1}$ replacing $x$ by $e^{i \pi} x$ and $e^{-i \pi} x$ respectively. This completes the evaluation of $\Phi_{1}$ for all finite values as well as for values near $\infty$ for all the parameter combinations given in Tables 2.1 and 2.2.

\section{Conclusion}

We have presented closed-form solutions of the non-relativistic linear perturbation equations which govern the evolution of inhomogeneities in a spatially flat multicomponent cosmological medium. The general solutions are catalogued according to the polytropic index $\gamma_{i}$ and the expansion law index $\eta$ of the multicomponent medium . All general solutions are expressed in terms of MEIJER's G-function and their MELLIN-BARNES integral representation. The proper use of this function simplifies the derivation of solutions of the linear perturbation equations and opens ways for its numerical computation. In this regard the paper leaves room for further work.

\section{Acknowledgments}

The authors would like to thank the Natural Sciences and the Engineering Research Councel of Canada for financial assistance for this research project. 


\section{References}

Bardeen, J.M.: 1980, Phys. Rev., D22, 1882

Haubold, H.J., Mathai, A.M., Muecket, J.P.: 1991, Astron. Nachr. 312,1

Hawking, S.W.: 1966, Ap.J., 145, 544

Lifshitz, E.M.: 1946, J. Phys. (Moscow), 10, 116

Lyth, D.H., Mukherjee, M.: 1988, Phys. Rev., D38, 485

Mathai, A.M.: 1989, Studies Appl. Math. 80,75.

Mathai, A.M., Saxena, R.K.: 1973. Generalized Hypergeometric Functions with Applications in Statistics and Physical Sciences. Lecture Notes in Mathematics, Vol. 348, Springer-Verlag, Berlin-Heidelberg-New York.

Nurgaliev, I.S.: 1986, Sov. Astron. Lett. 12, 73

Olson, D.W.: 1976, Phys. Rev., D14, 327

Peebles, P.J.E.: 1980, The Large-Scale Structure of the Universe, Princeton University Press, Princeton 